Eingriffe gemäß § 115 b SGB V aus Anhang 2 zu Kapitel 31 des EBM Stand 19. Jan. 2015

45. STIKO (2016) Empfehlungen der Ständigen Impfkommission (STIKO) am Robert Koch-Institut 2016/2017. Epidemiol Bull 34/2016:301-340 ((https://www.rki.de/DE/ Content/Infekt/EpidBull/Archiv/2016/Ausgaben/ 34_16.pdf?_blob=publicationFile))

46. Strittmatter B, Tabori E (2012) Koloproktologie. In: Zinn CG, Tabori E, Weidefeller P (Hrsg) Ambulantes Operieren - Praktische Hygiene. Verlag für medizinische Praxis, Pürgen

47. Strittmatter B, Tabori E (2017) Hygiene bei proktologischen Eingriffen. In: Mölle, Ommer, Lange, Girona (Hrsg) Chirurgische Proktologie, 3. Auflage

48. Sydnor ERM, Bova G, Gimburg A et al (2012) Electronic-eye faucets: legionella species contamination in healthcare settings. Infect Control Hosp Epidemiol 33(3):235-240

49. Tabori E (2012) Gynäkologie. In: Zinn GC, Tabori E, Weidenfeller P (Hrsg) Ambulantes Operieren Praktische Hygiene. Verlag für medizinische Praxis, Pürgen

50. Tabori E, Axmann S (2016) Infektionspräventive Anforderungen an bauliche Maßnahmen. Krankenhaushyg up2date 11:415-434. doi:10.1055/s0042-120781

51. Trinkwasserverordnung TrinkwV (2016) Verordnung über die Qualität von Wasser für den menschlichen Gebrauch (Trinkwasserverordnung 2001). Bundesgesetzblatt Jahrgang 2016 Teil I Nr. 12, ausgegeben zu Bonn am 16. März 2016. https://www.gesetze-im-internet.de/ bundesrecht/trinkwv_2001/gesamt.pdf (Erstellt: 18. Jul 2016). Zugegriffen: 21.02.2017

52. Weyandt GH (2010) Neue operative Ansätze zur Sanierung anogenitaler HPV-Infektionen. Hautarzt 62:28-33

53. Whaley S (2004) Tap water or normal saline for cleansing traumatic wounds? Br J Community Nurs 9(11):471-478

\title{
Erratum
}

coloproctology $2017 \cdot 39: 220$

DOI 10.1007/s00053-017-0159-y

Online publiziert: 13. April 2017

๑) Springer Medizin Verlag GmbH 2017

CrossMark

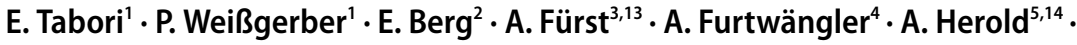
V. Kahlke ${ }^{6}$ B. Lenhard ${ }^{7} \cdot$ G. Osterholzer ${ }^{8,13} \cdot$ H. Peleikis ${ }^{6} \cdot$ T. Schiedeck $^{9,14}$. M. Schmidt-Lauber ${ }^{10,13} \cdot$ U. Schöffel ${ }^{11} \cdot$ M. Stoll ${ }^{12} \cdot$ B. Strittmatter ${ }^{4,13}$

'Deutsches Beratungszentrum für Hygiene (BZH), Freiburg, Deutschland

${ }^{2}$ Klinik für Koloproktologie, Prosper-Hospital, Recklinghausen, Deutschland

${ }^{3}$ Klinik für Chirurgie, Caritas-Krankenhaus St. Josef, Regensburg, Deutschland

${ }^{4}$ Praxisklinik 2000, Die Koloproktologen., Freiburg, Deutschland

${ }^{5}$ Enddarmzentrum Mannheim, Mannheim, Deutschland

${ }^{6}$ Proktologische Praxis Kiel, Kiel, Deutschland

${ }^{7}$ Praxis für Enddarmerkrankungen, Heidelberg, Deutschland

${ }^{8}$ Enddarmzentrum München-Bavaria, München, Deutschland

${ }^{9}$ Klinik für Allgemein- und Viszeralchirurgie, Klinikum Ludwigsburg, Ludwigsburg, Deutschland

${ }^{10}$ Gastroenterologische Gemeinschaftspraxis, Oldenburg, Deutschland

"Allgemein- und Viszeralchirurgie, Asklepios Klinik Lindau, Lindau, Deutschland

${ }^{12}$ End- und Dickdarmzentrum Hannover, Hannover, Deutschland

${ }^{13}$ Berufsverband der Coloproktologen Deutschlands e.V. (BCD), Freiburg, Deutschland

${ }^{14}$ Deutsche Gesellschaft für Koloproktologie e.V. (DGK), Freiburg, Deutschland

\section{Erratum zu: Leitfaden und Empfehlungen für die Hygiene in der Koloproktologie - Teil 1}

\section{Herausgegeben vom Berufsverband der Coloproktologen Deutschlands (BCD) und der Deutschen Gesellschaft für Koloproktologie (DGK)}

\section{Erratum zu: \\ coloproctology (2017) 39:111-120 \\ DOI 10.1007/s00053-017-0149-0}

In der Originalpublikation dieses Beitrags trat im Titel ein Fehler auf, wie auch in den Angaben zur Autorenschaft. Wir bitten, die korrekten Angaben zu beachten.

\section{Korrespondenzadresse}

\section{Dr. B. Strittmatter}

Berufsverband der Coloproktologen

Deutschlands e.V. (BCD)

Maienstraße 3, 79102 Freiburg, Deutschland info@coloproktologen.de 